\title{
PKS 1622-253: A WEAKLY ACCRETING, POWERFUL GAMMA-RAY SOURCE
}

\author{
BRIAN PUNSLY ${ }^{1}$ \\ International Center for Relativistic Astrophysics, Università degli Studi di Roma "La Sapienza," Piazzale Aldo Moro 5, I-00185 Rome, Italy; \\ brian.m.punsly@L-3com.com, brian.punsly@gte.net \\ LUIS F. RODRÍGUEZ \\ Centro de Radioastronomía y Astrofísica, Universidad Nacional Autónoma de México, Apdo. Postal 3-72 (Xangari), 58089 Morelia, \\ Michoacán, Mexico; 1.rodriguez@astrosmo.unam.mx \\ STEVEN J. TingaY \\ Centre for Astrophysics and Supercomputing, Swinburne University of Technology, P.O. Box 218, Hawthorn, \\ VIC 3122, Australia; stingay@astro.swin.edu.au \\ AND \\ Sergio A. Cellone \\ Facultad de Ciencias Astronómicas y Geofísicas, Universidad Nacional de La Plata, Paseo del Bosque, \\ B1900FWA La Plata, Argentina; scellone@fcaglp.unlp.edu.ar \\ Received 2005 August 29; accepted 2005 September 26; published 2005 October 19
}

\begin{abstract}
We discuss new deep radio observations of PKS 1622-253 and their implications for the energetics of the central engine that powers this strong high-energy gamma-ray source. Combining archival infrared and optical measurements with new millimeter observations, we show that even though the accretion flow in PKS 1622-253 is underluminous by quasar standards, a powerful superluminal jet is launched with a higher kinetic luminosity than most EGRET blazars. Only a few percent of the total jet kinetic luminosity is required to power even the most powerful gamma-ray flares that are observed. The implication is that a high-accretion system is not required in order to power the strongest high-energy gamma-ray sources.
\end{abstract}

Subject headings: accretion, accretion disks — black hole physics — galaxies: active — galaxies: jets quasars: general — quasars: individual (PKS 1622-253)

\section{INTRODUCTION}

The connection between accretion flow parameters and the gamma-ray luminosity from an associated radio jet is not well understood. Quasars are distinguished by the strong thermal UV luminosity associated with their accretion flows, and some in the blazar subcategory have been found to have strong apparent gamma-ray luminosities. Thus, it is of interest to understand why some of the stronger extragalactic sources identified by the Energetic Gamma-Ray Experiment Telescope (EGRET) have accretion flow luminosities below those associated with quasars (such as the FR II BL Lac object 0235+164, which has very weak optical emission in quiescent states; see Takalo et al. 1998). The discovery of a sizable class of strong gamma-ray sources with accretion flow luminosities less than typical for quasars would suggest that accretion flow luminosity is, at best, a secondary (indirect) physical factor in the regulation of gamma-ray emission from jets.

Motivated by this possibility, a study of the not so well known EGRET source PKS 1622-253 was initiated. Before the EGRET observations, this source was very rarely observed. However, it is one of the original EGRET detections and has been a firm identification in each of the three EGRET catalogs (Fichtel et al. 1994; Hartman et al. 1999). Part of the difficulty in observing this source is that it lies behind the dense cloud just south of $\rho$ Oph (Hunter et al. 1994). Hence, it is extremely faint in the optical (visual magnitude $m_{v}=21.9$ in the NASA/ IPAC Extragalactic Database, at $z=0.786$ ). Our primary findings are that only a small percentage of the jet kinetic luminosity is required to power the observed gamma-ray luminosity and that the accretion flow is underluminous by quasar standards.

\footnotetext{
${ }^{1}$ Postal address: 4014 Emerald Street, No. 116, Torrance, CA 90503.
}

\section{THE RADIO OBSERVATIONS}

New observations of PKS $1622-253$ at 6 and $3.6 \mathrm{~cm}$ were made on 2002 June 3 with the NRAO Very Large Array ${ }^{2}$ in the BnA configuration. The data were reduced using the standard VLA procedures and were self-calibrated in phase and amplitude as part of the imaging process. To obtain images at 6 and $3.6 \mathrm{~cm}$ of comparable angular resolution, the $6 \mathrm{~cm}$ image was made with the ROBUST parameter of IMAGR set to -5 , while the $3.6 \mathrm{~cm}$ image was made with the ROBUST parameter of IMAGR set to 5 and tapered in the $(u, v)$-plane to produce an angular resolution comparable to that of the $6 \mathrm{~cm}$ image. Finally, both images were restored with the same Gaussian beam. The morphologies of the images were similar, and we display the $6 \mathrm{~cm}$ radio map in Figure 1. To obtain the flux densities of the western and eastern lobes (indicated in Fig. 1), we subtracted a point source with the flux density and position of the core (also indicated in Fig. 1) from the $(u, v)$-data. Parameters of the core and lobes measured from the data are listed in Table 1. The two-point spectral index (calculated from 4.86 to $8.4 \mathrm{GHz}$ ) of the core clearly identifies it as distinct from the characteristically steep-spectrum lobes.

To estimate the flux densities of the lobes at a lower frequency, we used VLA archival data taken at $1.56 \mathrm{GHz}$ on 1991 October 13 in the A configuration. These results, also shown in Table 1, are not as reliable as those obtained at 6 and $3.6 \mathrm{~cm}$, since the lobes are only barely resolved from the core.

Some limited low-frequency radio data are available. PKS 1622-253 appears in the Molonglo Reference Catalogue

\footnotetext{
${ }^{2}$ The National Radio Astronomy Observatory is a facility of the National Science Foundation operated under cooperative agreement by Associated Universities, Inc.
} 


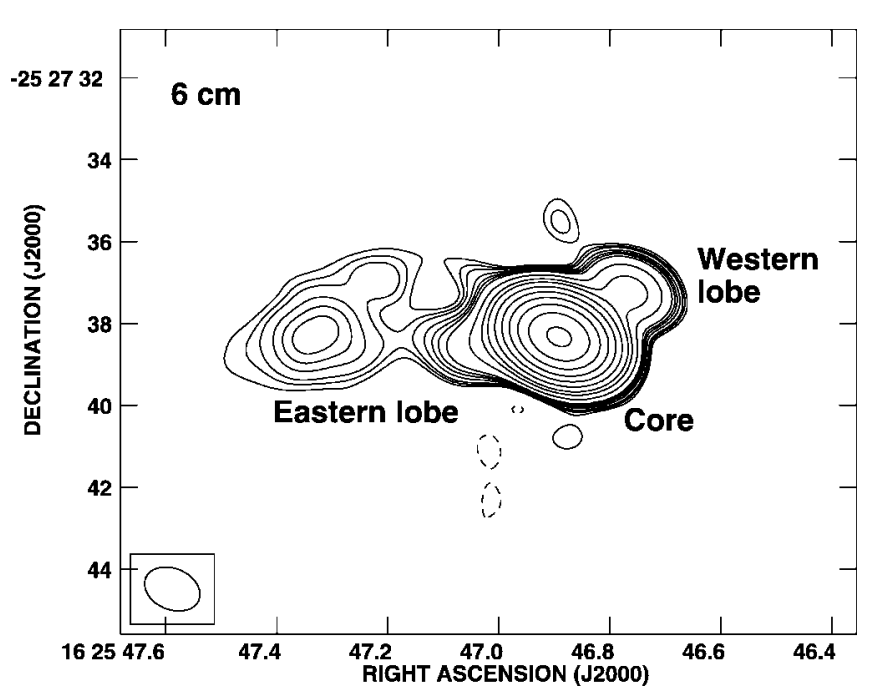

FIG. 1.-The $6 \mathrm{~cm}$ image of PKS $1622-253$. Note the powerful radio core that dominates the radio emission. There is a diffuse jetlike extension to the east and a very strong knot to the northwest that is apparently part of a jet that is a continuation of the parsec-scale jet that is directed at a similar position angle. The contours are $-4,4,5,6,8,10,12,15,30,60,100,200,400,800$, 1600,3200 , and 6400 times $0.25 \mathrm{mJy}$ beam $^{-1}$. The peak brightness of the $6 \mathrm{~cm}$ image is $1.84 \mathrm{Jy}_{\text {beam }}^{-1}$. The image is restored with a beam dimension of 1 ".40 41 1".0 and position angle of $67^{\circ}$. The main components of the source are labeled.

(Large et al. 1981) with a flux density of $2.36 \mathrm{Jy}$ at $408 \mathrm{MHz}$ and in the Texas Survey by Douglas et al. (1996) with a flux density of $2.352 \pm 0.047 \mathrm{Jy}$ at $365 \mathrm{MHz}$. While the optically thin lobe emission cannot be distinguished from the core at $\sim 400 \mathrm{MHz}$, an extrapolation of the VLA data shows that about $0.4 \mathrm{Jy}$ of lobe emission is present at this frequency (assuming that the core had approximately the same strength when the Molonglo and Texas observations were made).

\section{ESTIMATING THE JET KINETIC LUMINOSITY}

These maps can be used to determine the kinetic luminosity of the jet from a measurement of an isotropic (not affected by relativistic beaming) parameter, the strength of lobe emission. We take this to be the measured data in Table 1. In a superluminal source, such as PKS 1622-253, one must be extra cautious about fluxes' being greatly exaggerated by Doppler beaming, hence the need for an isotropic estimator such as optically thin lobe flux (Punsly 1995, 2005). In order to avoid the ambiguities associated with Doppler enhancement, we estimate the jet kinetic luminosity from the isotropic extended emission, applying a method that allows one to convert $151 \mathrm{MHz}$ flux densities, $F_{151}$, measured in janskys, into estimates of kinetic luminosity, $Q$, from Willott et al. (1999) and Blundell \& lings (2000). This estimator was expressed explicitly in terms of flux density in equations (1)-(2) of Punsly (2005):

$$
\begin{gathered}
Q \approx 1.1 \times 10^{45}\left[(1+z)^{1+\alpha} Z^{2} F_{151}\right]^{6 / 7} \operatorname{ergs~s}^{-1}, \\
Z \equiv 3.31-3.65\left[(1+z)^{4}-0.203(1+z)^{3}+0.749(1+z)^{2}\right. \\
+0.444(1+z)+0.205]^{-1 / 8}
\end{gathered}
$$

where $F_{151}$ is the total optically thin flux density from the lobes (i.e., no contribution from Doppler-boosted jets or radio cores). We assume a cosmology with $H_{0}=70 \mathrm{~km} \mathrm{~s}^{-1} \mathrm{Mpc}^{-1}, \Omega_{\Lambda}=$ 0.7 , and $\Omega_{m}=0.3$. In order to implement this technique, one
TABLE 1

Data Reduction from the Vla Observations

\begin{tabular}{lcccr}
\hline \hline Component & $\begin{array}{c}19 \mathrm{~cm} \\
(\mathrm{mJy})\end{array}$ & $\begin{array}{c}6 \mathrm{~cm} \\
(\mathrm{mJy})\end{array}$ & $\begin{array}{c}3.6 \mathrm{~cm} \\
(\mathrm{mJy})\end{array}$ & \multicolumn{1}{c}{$\begin{array}{c}\text { Spectral } \\
\text { Index }\end{array}$} \\
\hline Core $\ldots \ldots .$. & $1980 \pm 50$ & $1840 \pm 10$ & $1910 \pm 10$ & $-0.06 \pm 0.01$ \\
West $\ldots \ldots$. & $83 \pm 8$ & $35.6 \pm 0.9$ & $24.4 \pm 0.7$ & $0.64 \pm 0.07$ \\
East $\ldots \ldots$. & $145 \pm 15$ & $52.3 \pm 1.2$ & $35.1 \pm 1.1$ & $0.66 \pm 0.07$ \\
\hline
\end{tabular}

needs to determine which components are optically thin and which are Doppler enhanced.

One might suspect that the strong knot in the western component of Figure 1 is Doppler enhanced, since the parsec-scale jet is directed toward this feature with an apparent velocity of 14c (Jorstad et al. 2001; Tingay et al. 1998). However, the integrated flux of the western component is far weaker than that of the eastern component, and it is optically thin (see Table 1). Thus, we conclude that the western component is not likely to be significantly Doppler enhanced. We can fit the lobe flux densities in Table 1 with a two-component model, a strong knot with a spectral index $\alpha=0.58$ and a diffuse component with $\alpha=1.0$ from 1.56 to $8.46 \mathrm{GHz}$. The western and eastern components are respectively fitted with 10 and $20 \mathrm{mJy}$ of diffuse emission and 25.6 and $32.3 \mathrm{mJy}$ of knot emission at $4.86 \mathrm{GHz}$. This reproduces all of the average flux densities in Table 1 except for the $1.56 \mathrm{GHz}$ flux density, $125 \mathrm{mJy}$, of the eastern lobe, which falls short by $20 \mathrm{mJy}$. Extrapolating these power laws to $151 \mathrm{MHz}$ predicts $1.4 \mathrm{Jy}$ of lobe emission and, from equation (1), $Q \approx 1.7 \times 10^{45} \mathrm{ergs} \mathrm{s}^{-1}$. If one only considers the diffuse emission as significant at $151 \mathrm{MHz}$, one can obtain a conservative lower bound of $966 \mathrm{mJy}$, or $Q>1.2 \times 10^{45} \mathrm{ergs} \mathrm{s}^{-1}$.

For the sake of comparison, one can also use the isotropic estimator derived in equation (10) of Punsly (2005), which can be trivially adapted to $4.86 \mathrm{GHz}$,

$$
Q \approx \frac{\left[y_{1}(n)\right]^{(n-1) / 2}(486)^{\alpha}}{(n-2) a(n)} \times 10^{42}(1+z)^{1+\alpha} Z^{2} F_{4860} \operatorname{ergs~s}^{-1}+L,
$$

where the spectral index $\alpha=(n-1) / 2, L(\nu) \sim \nu^{-\alpha}, F_{4860}$ is the optically thin flux density at $4.86 \mathrm{GHz}$ in janskys, and the total radio luminosity is $L \equiv \int L(\nu) d \nu$. Table 1 indicates that the spectral index from 4.86 to $1.56 \mathrm{GHz}$ is $\alpha=0.83$. One can get $a(n)$ and $y_{1}(n)$ from Ginzburg (1979). Inserting these values into equation (2), with the aid of equation (1b) and Table 1 we find $Q \approx 1.29 \times 10^{45} \mathrm{ergs} \mathrm{s}^{-1}$. The close agreement between the two isotropic estimators is reassuring.

\section{ESTIMATING THE ACCRETION FLOW THERMAL LUMINOSITY}

The broadband continuum spectrum in Figure 2 (after correcting for the Galactic extinction from the Ophiuchus cloud using the results of Schlegel et al. 1998) indicates a broad synchrotron peak with a high-frequency tail with a spectral index of 1.4 that extends down to $2350 \AA$ in the quasar rest frame (di Serego Alighieri et al. 1994). Consistent with this interpretation is the significant polarization in the rest-frame near-UV, 2.8\% (Impey \& Tapia 1990).

Thus, one cannot detect the thermal signatures of the accretion flow. The best one can do is to obtain an estimate of the upper bound on the accretion disk luminosity, $L_{\mathrm{bol}}$. We tried to improve on this estimate by making our own optical obser- 
Spectrum of PKS 1622.253

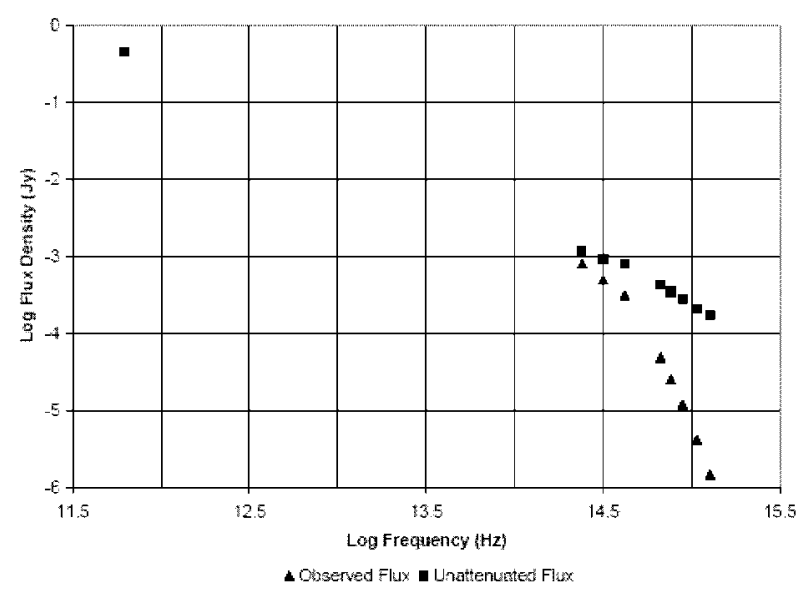

FIG. 2.-Continuum broadband spectrum of PKS 1622-253. The spectrum is not quasi-simultaneous. The lowest-frequency point is a millimeter observation courtesy of I. Robson, the three IR data points are from the Two Micron All Sky Survey, and the optical are from di Serego Alighieri et al. (1994). The triangles are the flux density as directly observed, and the squares are corrected for Galactic extinction. The frequency is expressed in the rest frame of the radio source.

vations of PKS 1622-253, hoping to catch the source in a low state of blazar activity and thereby improve the upper bound on the masked thermal emission. The source was observed on three consecutive nights with the $2.1 \mathrm{~m}$ Jorge Sahade Telescope at the Complejo Astronómico El Leoncito, Argentina, beginning in the evening of 2003 April 26 and ending in the morning of 2003 April 29; it was slightly brighter than when observed by di Serego Alighieri et al. (1994) and therefore was too bright for these observations to improve our knowledge of the accretion disk luminosity.

One can estimate $L_{\text {bol }}$ by noting that the bolometric luminosity of the thermal emission from the accretion flow includes optical, UV, and any radiation in broad emission lines from photoionized gas or as IR reprocessed by molecular gas. In order to place an upper bound on $L_{\text {bol }}$, we construct a composite spectral energy distribution (SED) of a quasar accretion flow. In order to separate the accretion flow's thermal luminosity from IR and optical contamination from the jet, a SED for radio-quiet quasars was chosen, since this represents pure accretion luminosity. In radio-quiet quasars, weak versions of radio-loud jets apparently exist (Barvainis et al. 2005). The bolometric luminosity of radio-quiet quasars is dominated by IR-to-UV emission, that is, the radio emission from the jet does not contribute significantly to the bolometric luminosity (Laor 1998). Furthermore, the IR synchrotron emission from the jet is swamped by thermal dust emission in radio-quiet quasars (Haas et al. 1998, 2000). Thus, the IR-to-X-ray composite of a radio-quiet quasar seems to represent the thermal emission from a viscous accretion flow. A piecewise collection of power laws is used to approximate the individual bands of a radioquiet quasar accretion flow luminosity in the composite SED. The IR and optical data are from the composite spectrum of Elvis et al. (1994), the near-UV and extreme-UV data are from the Hubble Space Telescope composites of Zheng et al. (1997) and Telfer et al. (2002), and the X-ray portion of the composite is from Laor et al. (1997). One can compute the bolometric luminosity of the accretion flow from the composite SED. In addition to the continuum, the composite spectrum of Zheng et al. (1997) indicates that $\approx 25 \%$ of the total optical/UV quasar luminosity is reprocessed in the broad-line region. Combining this with the continuum luminosity yields $L_{\text {bol }}=1.35$ $\times 10^{46} \mathrm{ergs} \mathrm{s}^{-1}$. The absolute visual magnitude of the composite is $M_{V}=-25$. Comparing the $2350 \AA$ spectral luminosity of PKS 1622-253 from Figure 2, $\nu F_{\nu}<3 \times 10^{45} \mathrm{ergs} \mathrm{s}^{-1}$, with the value from the composite $\mathrm{SED}, \nu F_{\nu}=4 \times 10^{45} \mathrm{ergs} \mathrm{s}^{-1}$, one finds an upper bound of $L_{\text {bol }}<10^{46} \mathrm{ergs} \mathrm{s}^{-1}$ for PKS $1622-253$. However, since there is no deviation from the steep power law in the spectrum of di Serego Alighieri et al. (1994) at high frequency, we claim that the actual value of $L_{\text {bol }}$ from an underlying accretion disk is far less than this. One way to obtain a crude estimate of $L_{\text {bol }}$ when the blazar jet masks the thermal UV flux is to use a broad-line luminosity. For example, Wang et al. (2004) used the composite spectrum of Francis et al. (1991) to motivate the relation $L_{\mathrm{bol}} \approx 252.6 L_{\mathrm{H} \beta}$, where $L_{\mathrm{H} \beta}$ is the luminosity of the $\mathrm{H} \beta$ broad emission line. Using the line strength from di Serego Alighieri et al. (1994) and correcting for Galactic extinction using the results of Schlegel et al. (1998), we find $L_{\text {bol }} \approx 2.19 \times 10^{45} \mathrm{ergs} \mathrm{s}^{-1}$.

If one also assumes that the shape of the composite spectrum is independent of quasar luminosity, a simple approximate formula is obtained that relates the $k$-corrected absolute visual magnitude to bolometric luminosity,

$$
L_{\mathrm{bol}} \approx\left(1.35 \times 10^{-\left(25+M_{V}\right) / 2.5}\right) \times 10^{46} \mathrm{ergs} \mathrm{s}^{-1} .
$$

Thus, subtracting out the optical emission from the jet, the estimate of $L_{\text {bol }}$ above suggests that the accretion flow alone would produce $M_{V}=-23$, right at the typical dividing line between Seyfert 1's and quasars (Véron-Cetty \& Véron 2001).

\section{DISCUSSION}

We performed deep radio observations of PKS 1622-253 with the VLA and also extricated the intrinsic IR/optical spectrum from the attenuation due to the Ophiuchus cloud. Our conclusion is that this source is a classical triple radio galaxy with a powerful superluminal radio core and powerful FR II radio lobes. The optical/near-UV emission is dominated by the high-frequency tail of a very broad synchrotron spectrum associated with the powerful radio core. The intrinsic accretion flow luminosity is at the quasar-Seyfert 1 dividing line, $M_{V}=-23$ (Véron-Cetty \& Véron 2001). It was shown that PKS 1622-253 has roughly equal jet kinetic luminosity and accretion-flow bolometric luminosity, $\gtrsim 10^{45} \mathrm{ergs} \mathrm{s}^{-1}$.

The gamma-ray apparent luminosity has a time-average value of $\sim 10^{47} \mathrm{ergs} \mathrm{s}^{-1}$ and flares at $\sim 10^{48} \mathrm{ergs} \mathrm{s}^{-1}$ (Hartman et al. 1999). The gamma-ray luminosity is enhanced by the Doppler factor to the fourth power, $\delta^{4}$. In order to determine the intrinsic gamma-ray luminosity of the jets, one must know the Doppler factor of the high-energy plasma. The best estimates we have are from measurements of the apparent superluminal jet speeds on VLBI scales. This scale is much larger than the gamma-ray-emitting region, and it is not clear whether one is observing pattern or bulk-flow speeds. Higher resolution typically yields higher apparent speeds (Kellermann et al. 2004). Thus, the maximal apparent superluminal velocity from VLBI for each blazar is probably the best estimator of the Doppler factor of the gamma-ray plasma. The superluminal motion and the variability results of Kellermann et al. (2004), and the beaming analysis of Padovani \& Urry (1992), indicate 
that strong blazar jets in quasars are typically enhanced by $10^{4}<\delta^{4}<10^{5}$. The extremely superluminal jet speed of $14 c$ (Jorstad et al. 2001) and the extreme radio flux and radio polarization variability (Tingay et al. 2003) imply that PKS $1622-253$ is an extreme blazar and $\delta^{4} \gtrsim 10^{4}$ is likely in flare states. Thus, even during a gamma-ray flare less than $10 \%$ of the intrinsic jet kinetic luminosity is needed to power the gamma-ray emission, and on average less than $1 \%$ of the intrinsic jet energy is converted into gamma rays.

Finally, we note that such powerful jets from weakly accreting systems are not rare for EGRET sources. The most extreme case is PKS $0202+149$, at $z=0.405$ with $m_{v}=22.1$ and a very steep optical spectral index (Perlman et al. 1998). Applying the methods of $\S 4$, one concludes that $L_{\text {bol }}<10^{44} \mathrm{ergs} \mathrm{s}^{-1}$. It does not have FR II radio lobes (or any extended radio emission for that matter; see Murphy et al. 1993), so one cannot make an isotropic estimate of the jet kinetic luminosity. However, it has been observed more than once with an apparent gamma-ray luminosity over $10^{47} \mathrm{ergs} \mathrm{s}^{-1}$ (Hartman et al. 1999). Apparently, strong jets can be driven without a strong accretion flow, and if there are also large Doppler factors associated with a nearly line-of-sight jet, gamma-ray fluxes detectable by EGRET can be achieved. A plausible theoretical explanation is provided in Punsly (2001), in which it is claimed that it is the accumulation of vertical magnetic flux near the black hole that is the primary determinant of jet power in FR II radio sources, not the accretion flow proper. The release of black hole spin energy, in the form of a relativistic jet, by the largescale torque applied by the magnetic flux is clearly shown, in accord with the theory, in the simulations of Semenov et al. (2004).

S. A. C. acknowledges financial support from the Agencia Nacional de Promoción Científica y Tecnológica of Argentina (PICT 03-13291) granted to Gustavo Romero.

\section{REFERENCES}

Barvainis, R., Lehár, J., Birkinshaw, M., Falcke, H., \& Blundell, K. M. 2005, ApJ, 618, 108

Blundell, K. M., \& Rawlings, S. 2000, AJ, 119, 1111

di Serego Alighieri, S., Danziger, I. J., Morganti, R., \& Tadhunter, C. N. 1994, MNRAS, 269, 998

Douglas, J. N., Bash, F. N., Bozyan, F. A., Torrence, G. W., \& Wolfe, C. 1996, AJ, 111, 1945

Elvis, M., et al. 1994, ApJS, 95, 1

Fichtel, C., et al. 1994, ApJS, 94, 551

Francis, P., Hewett, P. C., Foltz, C. B., Chaffee, F. H., Weymann, R. J., \& Morris, S. L. 1991, ApJ, 373, 465

Ginzburg, V. L. 1979, Theoretical Physics and Astrophysics, transl. D. ter Haar (New York: Pergamon)

Haas, M., Chini, R., Meisenheimer, K., Stickel, M., Lemke, D., Klaas, U., \& Kreysa, E. 1998, ApJ, 503, L109

Haas, M., Müller, S. A. H., Chini, R., Meisenheimer, K., Klaas, U., Lemke, D., Kreysa, E., \& Camenzind, M. 2000, A\&A, 354, 453

Hartman, R. C., et al. 1999, ApJS, 123, 79

Hunter, S. D., Digel, S. W., de Geus, E. J., \& Kanbach, G. 1994, ApJ, 436, 216

Impey, C. D., \& Tapia, S. 1990, ApJ, 354, 124

Jorstad, S. G., Marscher, A. P., Mattox, J. R., Wehrle, A. E., Bloom, S. D., \& Yurchenko, A. V. 2001, ApJS, 134, 181

Kellermann, K. I., et al. 2004, ApJ, 609, 539

Laor, A. 1998, ApJ, 505, L83
Laor, A., Fiore, F., Elvis, M., Wilkes, B. J., \& McDowell, J. C. 1997, ApJ, 477,93

Large, M. I., Mills, B. Y., Little, A. G., Crawford, D. F., \& Sutton, J. M. 1981, MNRAS, 194, 693

Murphy, D., Browne, I. W. A., \& Perley, R. A. 1993, MNRAS, 264, 298

Padovani, P., \& Urry, C. M. 1992, ApJ, 387, 449

Perlman, E. S., Padovani, P., Giommi, P., Sambruna, R., Jones, L. R., Tzioumis, A., \& Reynolds, J. 1998, AJ, 115, 1253

Punsly, B. 1995, AJ, 109, 1555 2001, Black Hole Gravitohydromagnetics (New York: Springer) 2005, ApJ, 623, L9

Schlegel, D. J., Finkbeiner, D. P., \& Davis, M. 1998, ApJ, 500, 525

Semenov, V., Dyadechkin, S., \& Punsly, B. 2004, Science, 305, 978

Takalo, L. O., et al. 1998, A\&AS, 129, 577

Telfer, R. C., Zheng, W., Kriss, G. A., \& Davidsen, A. F. 2002, ApJ, 565, 773

Tingay, S. J., Jauncey, D. L., King, E. A., Tzioumis, A. K., Lovell, J. E. J., \& Edwards, P. G. 2003, PASJ, 55, 351

Tingay, S. J., Murphy, D. W., \& Edwards, P. G. 1998, ApJ, 500, 673

Véron-Cetty, M.-P., \& Véron, M. 2001, A\&A, 374, 92

Wang, J.-M., Luo, B., \& Ho, L. C. 2004, ApJ, 615, L9

Willott, C. J., Rawlings, S., Blundell, K. M., \& Lacy, M. 1999, MNRAS, 309, 1017

Zheng, W., Kriss, G. A., Telfer, R. C., Grimes, J. P., \& Davidsen, A. F. 1997, ApJ, 475, 469 